\title{
Magnetite Schottky barriers on GaAs substrates
}

\author{
Steven M. Watts, Catherine Boothman, Sebastiaan van Dijken, ${ }^{\text {a) }}$ and J. M. D. Coey \\ SFI Trinity Nanoscience Laboratory, Physics Department, Trinity College, Dublin 2, Ireland
}

(Received 31 January 2005; accepted 13 April 2005; published online 20 May 2005)

\begin{abstract}
Carrier transport across $\mathrm{Fe}_{3} \mathrm{O}_{4} / \mathrm{GaAs}$ interfaces has been studied for $n$ - and $p$-type GaAs(001) substrates with medium $\left(7.7 \times 10^{17} \mathrm{~cm}^{-3}\right)$ to high $\left(3.5 \times 10^{18} \mathrm{~cm}^{-3}\right)$ carrier concentrations. Currentvoltage $(I-V)$ measurements on medium-doped substrates show a rectifying behavior that is characteristic for thermionic emission/diffusion across a Schottky barrier. The $n$-type structure exhibits a low ideality factor of 1.3 and a Schottky barrier height of $0.58-0.63 \mathrm{eV}$. The Schottky barrier height of the $p$-type sample is $0.51 \mathrm{eV}$. For $\mathrm{Fe}_{3} \mathrm{O}_{4} / \mathrm{GaAs}$ structures with higher doping levels the $I-V$ dependence is nearly symmetric. In this case, tunneling of electrons and holes through the Schottky barrier dominates transport between the $\mathrm{Fe}_{3} \mathrm{O}_{4}$ layer and the GaAs substrate. (C) 2005 American Institute of Physics. [DOI: 10.1063/1.1925758]
\end{abstract}

Electrical spin injection and detection has been demonstrated between ferromagnetic and normal metals ${ }^{1}$ and in semiconductor/(magnetic) semiconductor heterostructures., ${ }^{2,3}$ However, one of the main goals of spin electronics, to achieve spin injection from a ferromagnetic metal into a semiconductor, has been more difficult to attain. One particular obstacle is the conductivity mismatch between metals and semiconductors, which drastically limits the spin injection efficiency for ohmic contacts between metals and semiconductors. ${ }^{4}$ For nonohmic contacts, however, the conductivity mismatch model of Schmidt et al. does not apply and it has recently been demonstrated that spin injection can be obtained by tunneling through a barrier at the semiconductor interface ${ }^{5}$ or by using a magnetic tunnel transistor emitter. $^{6}$

An alternative route for spin injection is to use a halfmetallic ferromagnet, which has a completely spin-polarized conduction band, as the spin injector. For perfectly spinpolarized sources, the conductivity mismatch is circumvented even for ohmic contacts, and in the case of tunneling the higher polarization should give a much larger spin accumulation in the semiconductor compared to $3 d$ transition metal injectors. Unfortunately the growth of half-metallic ferromagnets onto semiconductors tends to be more difficult than ferromagnetic transition metals, and very often the spin polarization will be determined more by disordered interfacial layers than by the bulk properties of the ferromagnet itself. Furthermore, perfect spin polarization is a zerotemperature property. The two spin states are mixed, to some extent, at finite temperature. Nonetheless, $\mathrm{Fe}_{3} \mathrm{O}_{4}$ has a number of unusual properties that make it attractive as a spinpolarized source.

As a class $\mathrm{II}_{\mathrm{B}}$ half-metal, $\mathrm{Fe}_{3} \mathrm{O}_{4}$ is not truly a metal but it is rather a polaronic hopping conductor of minority spins. ${ }^{7}$ The resistivity increases with decreasing temperature and goes up sharply at the Verwey transition at about $120 \mathrm{~K}{ }^{8}$ Thus, for ohmic contacts, the resistance match between $\mathrm{Fe}_{3} \mathrm{O}_{4}$ and the semiconductor may be tuned by adjusting the temperature. For Schottky tunnel barrier contacts, there is the advantage of a completely spin-polarized density of states at the Fermi level.

\footnotetext{
${ }^{a)}$ Electronic mail: vandijks@tcd.ie
}

Here, we show that high-quality Schottky diodes can be obtained by depositing the half-metallic hopping conductor $\mathrm{Fe}_{3} \mathrm{O}_{4}$ onto $n$ - and $p$-type GaAs substrates. In addition, measurements on GaAs substrates with different carrier concentrations reveal that different transport modes can be selected by proper engineering of the semiconductor doping profile.

The $\mathrm{Fe}_{3} \mathrm{O}_{4}$ films were deposited onto $\mathrm{GaAs}(001)$ substrates at $400{ }^{\circ} \mathrm{C}$ by reactive dc sputtering of a pure Fe target. Partial $\mathrm{Ar}$ and $\mathrm{O}_{2}$ pressures during growth were 3 $\times 10^{-3}$ mbar and $4 \times 10^{-5} \mathrm{mbar}$, respectively. This deposition procedure resulted in a preferential (111) film texture. Magnetization and in-plane transport measurements showed a clear Verwey transition at about $120 \mathrm{~K} .^{9}$ The carrier concentrations of the four different GaAs substrates as determined by Hall effect measurements were $7.7 \times 10^{17} \mathrm{~cm}^{-3}$ (medium $n$ type), $1.0 \times 10^{18} \mathrm{~cm}^{-3}$ (medium $p$ type), 3.5 $\times 10^{18} \mathrm{~cm}^{-3}$ (high $n$ type), and $3.4 \times 10^{18} \mathrm{~cm}^{-3}$ (high $p$ type) For the current-voltage $(I-V)$ measurements, an ohmic contact was made to the back of the GaAs substrate by thermal evaporation of an $\mathrm{Au}-\mathrm{Ge}$ alloy and brief annealing at $300{ }^{\circ} \mathrm{C}$ in air. A layer of Au was thermally evaporated onto the magnetite surface to form the top contact. In the results reported below, the thickness of the magnetite layer was about $60 \mathrm{~nm}$ and the junction area was typically $40 \mathrm{~mm}^{2}$. The temperature-dependent measurements were made in a closed-cycle helium refrigerator system.

Figure 1 shows the $I-V$ curves for the four different GaAs substrates at $50 \mathrm{~K}$ intervals between $50 \mathrm{~K}$ and $300 \mathrm{~K}$. For the $n$ - and $p$-type samples with a medium carrier concentration, the data are asymmetric indicating a diodelike behavior that is typical for Schottky barriers. The mediumdoped $n$-type substrate has the lowest doping level and, consequently, the leakage current in reverse bias is smallest for this sample. The structures with higher doping levels show nearly ohmic characteristics at room temperature, but significant deviation from linearity at lower temperatures.

For the medium-doped substrates, transport across the $\mathrm{Fe}_{3} \mathrm{O}_{4} / \mathrm{GaAs}$ interface is dominated by thermionic emission/ diffusion at elevated temperatures. In the thermionic emission/diffusion model, ${ }^{10}$ the dependence of the current on bias voltage is expressed as 


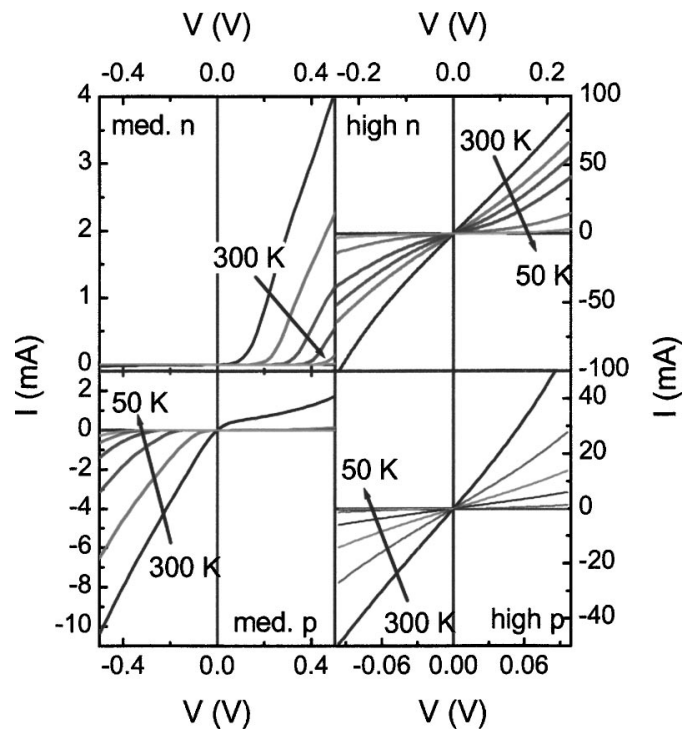

FIG. 1. (Color online) $I-V$ curves for $\mathrm{Fe}_{3} \mathrm{O}_{4} / \mathrm{GaAs}$ structures with medium and high GaAs carrier concentrations. The top and bottom graphs show results for $n$-type and $p$-type GaAs, respectively. Data are shown for temperatures between $50 \mathrm{~K}$ and $300 \mathrm{~K}$ at increments of $50 \mathrm{~K}$.

$$
I=I_{S}\left(e^{q V / n k T}-1\right) .
$$

Here, $I_{S}=A_{e} A^{* *} T^{2} e^{q \phi / k T}, A_{e}$ is the active area, $A^{* *}$ is the effective Richardson constant, $\phi$ is the Schottky barrier height, and $n$ is the ideality factor. Differentiating and taking the logarithm gives

$$
\ln (d I / d V)=\ln \left(I_{S} q / n k T\right)+q V / n k T .
$$

Thus the slope of the data plotted as $\ln (d I / d V)$ versus $V$ will directly give the ideality factor and the intercept can be used to calculate the Schottky barrier height. Figure 2 shows the data for the medium-doped samples after numerical differentiation, plotted in this way.

For the medium-doped $n$-type substrate the data for $T$ $>230 \mathrm{~K}$ can be fitted between zero bias and a forward bias of about $0.1 \mathrm{~V}$, above which the current saturates due to series resistance. Near room temperature, an ideality factor

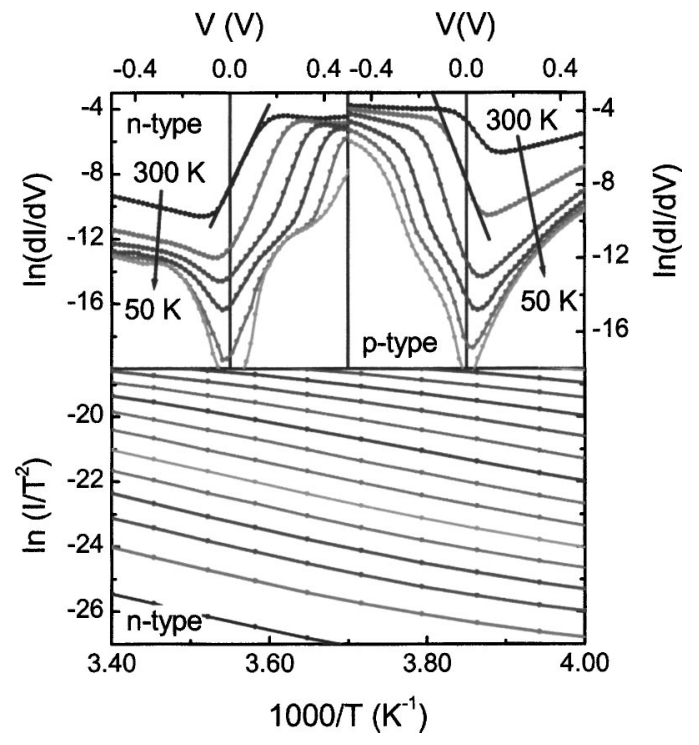

FIG. 2. (Color online) Logarithm of the conductance versus bias voltage for the medium-doped $n$ - and $p$-type samples (top) and activation energy plots

for the medium-doped $n$-type sample (bottom). calculated and experimentally determined value of $I_{S}$ are
Downloaded 12 Aug 2009 to 134.226 .1 .229 . Redistribution subject to AlP license or copyright; see http://apl.aip.org/apl/copyright.jsp $n=1.3$ and a Schottky barrier height $q \phi=0.63 \mathrm{eV}$ are obtained. To determine $\phi$, we used the total area of the sample (approximately $40 \mathrm{~mm}^{2}$ ) and an effective Richardson constant of $8.2 \mathrm{~A} \mathrm{~cm}^{-2} \mathrm{~K}^{-2} \cdot{ }^{10}$ Alternatively, the Schottky barrier height can be obtained from an activation energy plot. In this case, it is not necessary to specify $A_{e}$ and $A^{* *}$, which is an advantage for large junction areas. In our analysis, we use

$$
\ln \left(\mathrm{I} / T^{2}\right)=\ln \left(A_{e} A^{* *}\right)-\frac{q(\phi-V / n)}{k T} .
$$

The activation energy plots for different forward biases near room temperature are shown in the lower part of Fig. 2. From the slope of the high-temperature data $(260 \leqslant T$ $\leqslant 300)$ and $n=1.3$, we obtain $q \phi=0.58 \mathrm{eV}$, which is slighter smaller than the result from the previous analysis.

For the medium-doped $p$-type substrate, a series resistance limits the current in forward bias at high temperature. As a result, Eq. (2) cannot be used to estimate the Schottky barrier height. Between $200 \mathrm{~K}$ and $250 \mathrm{~K}$, however, the Schottky barrier resistance dominates the series resistance. A thermionic emission/diffusion analysis in this temperature range yields $n=1.3$ and $q \phi=0.51 \mathrm{eV}$.

The $n$ - and $p$-type GaAs substrates with higher doping concentrations exhibit similar $I-V$ characteristics that are nearly symmetric with voltage and clearly nonlinear at lower temperatures. In this case, carrier transport across the $\mathrm{Fe}_{3} \mathrm{O}_{4} / \mathrm{GaAs}$ interface is dominated by tunneling of electrons or holes through the Schottky barrier. The data can be analyzed within the (thermionic) field emission model of Padovani and Stratton. ${ }^{11}$ In this model, the $I-V$ dependence is expressed as

$$
I=I_{S} e^{\left(q V / E_{0}\right)},
$$

where $I_{S}$ is the saturation current and $E_{0}$ is an energy term that depends on bias voltage and temperature. For an intermediate temperature regime in which thermionic field emission dominates, $I_{S}$ and $E_{0}$ are given by

$$
\begin{aligned}
& I_{S}=\frac{A_{e} A^{*} \sqrt{\pi E_{00}\left(\phi-q V+E_{\mathrm{fsc}}\right)} \exp \left(\frac{E_{\mathrm{fsc}}}{k T}-\frac{\phi+E_{\mathrm{fsc}}}{E_{0}}\right)}{k T \cosh \left(E_{00} / k T\right)}, \\
& E_{0}=E_{00} \operatorname{coth}\left(E_{00} / k T\right) .
\end{aligned}
$$

Here, $E_{00}=q \hbar / 4 \sqrt{N / \varepsilon m^{*}}$ and $E_{\mathrm{fsc}}$ is the Fermi level of the semiconductor. Similar expressions have been derived for reverse bias voltages. ${ }^{11}$ At low temperatures, field emission dominates transport across the metal/semiconductor interface and $E_{0}=E_{00}$. We fitted the $I-V$ characteristics of the $n$ - and $p$-type GaAs substrates with high doping concentrations with

$$
V=E_{0}\left(\ln I-\ln I_{S}\right)+I R_{S},
$$

where $R_{S}$ is introduced to account for a series resistance. The results are shown in Fig. 3 together with the theoretical predictions for $I_{S}$ and $E_{0}$ for a forward-biased $n$-type substrate with $n=3.5 \times 10^{18} \mathrm{~cm}^{-3}$ (based on textbook parameters for GaAs). ${ }^{10}$ The experimentally determined values for $I_{S}$ and $E_{0}$ are of the same order of magnitude as the calculated ones. Whereas the predicted value of $E_{0}$ shows only minor temperature dependence, the temperature dependence of both the calculated and experimentally determined value of $I_{S}$ are AIP license or copyright; see http://apl.aip.org/apl/copyright.jsp 


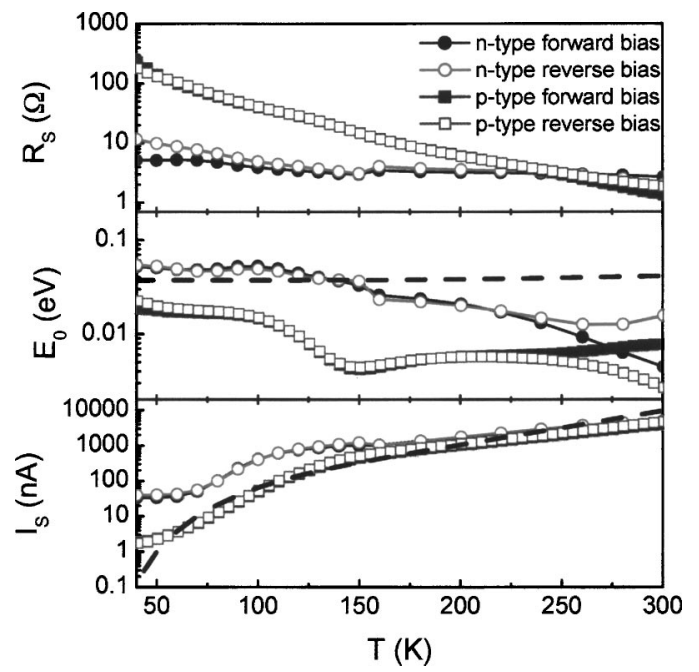

FIG. 3. (Color online) Temperature dependence of $R_{S}, E_{0}$, and $I_{S}$. These data were extracted by fitting the experimental data of the $n$ - and $p$-type GaAs substrates with high carrier concentration with Eq. (7) $\left(V_{\text {bias }}=10 \mathrm{mV}\right)$. The dashed line represents a calculation for the $n$-type substrate with a high carrier concentration of $3.5 \times 10^{18} \mathrm{~cm}^{-3}$ at a forward bias of $10 \mathrm{mV}$.

qualitatively similar above $80 \mathrm{~K}$. This confirms that transport across the $\mathrm{Fe}_{3} \mathrm{O}_{4} / \mathrm{GaAs}$ interface is dominated by (thermionic) field emission for the higher-doped substrates.

The series resistance $R_{S}$ of the $\mathrm{Fe}_{3} \mathrm{O}_{4} / \mathrm{GaAs}$ structures increases with decreasing temperature. This effect, which is most pronounced for the $p$-type substrate, is due to the temperature dependence of the $\mathrm{Fe}_{3} \mathrm{O}_{4}$ layer and $\mathrm{GaAs}$ substrate resistances. While the resistance of the $\mathrm{Fe}_{3} \mathrm{O}_{4}$ layer increases with decreasing temperature, the variation of the GaAs resistance can either be positive or negative depending on the doping level. ${ }^{10}$ In addition, the $\mathrm{Fe}_{3} \mathrm{O}_{4}$ layer can also contribute to the nonlinearity in the $I-V$ curves at low temperatures. Current out-of-plane measurements on $\mathrm{Au} / \mathrm{Fe}_{3} \mathrm{O}_{4} / \mathrm{Au}$ trilayer structures reveal a nonlinear $I-V$ dependence below the Verwey transition. ${ }^{12}$ This effect is most likely due to the opening of a gap in the minority spin band below $120 \mathrm{~K}$, which creates an additional transport barrier at the $\mathrm{Au} / \mathrm{Fe}_{3} \mathrm{O}_{4}$ interface.

In conclusion, we have identified different transport mechanisms across $\mathrm{Fe}_{3} \mathrm{O}_{4} / \mathrm{GaAs}$ interfaces. For $n$ - and $p$-type GaAs substrates with medium doping concentrations, we measured a rectifying behavior that is characteristic of thermionic emission/diffusion across a Schottky barrier. Fits to the data reveal $n=1.3$ and $\phi=0.58-0.63 \mathrm{eV}$ for a $n$-type carrier concentration of $7.7 \times 10^{17} \mathrm{~cm}^{-3}$ and $n=1.3$ and $\phi=0.51 \mathrm{eV}$ for a $p$-type carrier concentration of 1.0 $\times 10^{18} \mathrm{~cm}^{-3}$. This demonstrates that it is possible to form a high-quality Schottky barrier at the interface of the halfmetallic hopping conductor $\mathrm{Fe}_{3} \mathrm{O}_{4}$ and GaAs. For higher doping concentrations $\left(n, p \approx 3.5 \times 10^{18} \mathrm{~cm}^{-3}\right)$, transport across the $\mathrm{Fe}_{3} \mathrm{O}_{4} / \mathrm{GaAs}$ interface is dominated by (thermionic) field emission. In this case, electrons and holes tunnel through the Schottky barrier and this results in nonlinear but nearly symmetric $I-V$ curves. Our data show that different transport modes between a half-metal and a semiconductor can be selected by proper engineering of the semiconductor doping profile. This is promising for the application of $\mathrm{Fe}_{3} \mathrm{O}_{4}$ as a spin-polarized source, which is expected to further enhance the polarization of the current in spin injection experiments.

This work was supported by Science Foundation Ireland as part of the CINSE program. We would like to acknowledge S. von Molnár and J. Trbovic for helpful discussions.

${ }^{1}$ F. J. Jedema, A. T. Filip, and B. J. van Wees, Nature (London) 410, 345 (2001); F. J. Jedema, H. B. Heersche, A. T. Filip, J. J. A. Baselmans, and B. J. van Wees, ibid. 416, 713 (2002).

${ }^{2}$ R. Fiederling, M. Keim, G. Reuscher, W. Ossau, G. Schmidt, A. Waag, and L. W. Molenkamp, Nature (London) 402, 787 (1999).

${ }^{3}$ Y. Ohno, D. K. Young, B. Beschoten, F. Matsukura, H. Ohno, and D. D. Awschalom, Nature (London) 402, 790 (1999).

${ }^{4}$ G. Schmidt, D. Ferrand, L. W. Molenkamp, A. T. Filip, and B. J. van Wees, Phys. Rev. B 62, R4790 (2000).

${ }^{5}$ A. T. Hanbicki, O. M. J. van't Erve, R. Magno, G. Kioseoglou, C. H. Li, and B. T. Jonker, Appl. Phys. Lett. 82, 4092 (2003); O. M. J. van't Erve, G. Kioseoglou, A. T. Hanbicki, C. H. Li, B. T. Jonker, R. Mallory, M. Yasar, and A. Petrou, ibid. 84, 4334 (2004).

${ }^{6}$ X. Jiang, R. Wang, S. van Dijken, R. Shelby, R. Macfarlane, G. S. Solomon, J. Harris, and S. S. P. Parkin, Phys. Rev. Lett. 90, 256603 (2003).

${ }^{7}$ J. M. D. Coey and M. Venkatesan, J. Appl. Phys. 91, 8345 (2002).

${ }^{8}$ F. Walz, J. Phys.: Condens. Matter 14, R285 (2002).

${ }^{9}$ S. M. Watts, K. Nakajima, S. van Dijken, and J. M. D. Coey, J. Appl. Phys. 95, 7465 (2004).

${ }^{10}$ S. M. Sze, Physics of Semiconductor Devices, 2nd ed. (Wiley, New York, 1981).

${ }^{11}$ F. A. Padovani and R. Stratton, Solid-State Electron. 9, 695 (1966).

${ }^{12}$ S. M. Watts, S. Blanc, S. van Dijken, and J. M. D. Coey (unpublished). 